\title{
Changes in the use of Otorhinolaryngology Emergency Department during the COVID-19 pandemic: report from Lombardy, Italy
}

\author{
Fabrizia Elli ${ }^{1}$ - Mario Turri-Zanoni ${ }^{1,2} \cdot$ Alberto Daniele Arosio $^{1} \cdot$ Apostolos Karligkiotis $^{1} \cdot$ Paolo Battaglia $^{1,2}$. \\ Paolo Castelnuovo ${ }^{1,2}$
}

Received: 19 May 2020 / Accepted: 8 June 2020 / Published online: 11 June 2020

(c) Springer-Verlag GmbH Germany, part of Springer Nature 2020

\begin{abstract}
Purpose This paper aims to report the impact of COVID-19 outbreak on Emergency Ear nose and Throat (ENT) activity, in Lombardy (Italy), during COVID-19 pandemic.

Methods The number of accesses to Emergency Department requiring ENT consultations between February 21st and May 7th, 2020, was retrospectively collected, along with the conditions treated. Data were stratified in 1-week time spans, to evaluate the evolving trends during the on-going epidemic and a comparison with the two previous years was performed.

Results A significant reduction in the number of consultations performed during the pandemic was observed, as high as $-91 \%$ compared to the same period of 2018 .

Conclusion Multiple reasons can explain such a reduction of Emergency ENT consultations, not least the fear of potentially being infected by SARS-CoV-2 by while accessing the hospital. The analysis performed might be useful as a starting point for a future reorganization of first aid consultations once the epidemic will be resolved.
\end{abstract}

Keywords COVID 19 $\cdot$ Outpatients $\cdot$ Endoscopy $\cdot$ Emergency Department $\cdot$ ENT

\section{Introduction}

The SARS-CoV-2 pandemic affected over 6 million cases causing more than 350,000 deaths globally up to the end of May 2020 [1]. Italy was the first-hit European country, now counting more than 230,000 SARS-CoV-2-positive cases and over 33,000 deaths [1]. Initially, uncertainty and underestimation of the disease created an unexpected situation that was accompanied by confusion and early disorganization.

MTZ is a PhD student on the "Biotechnologies and Life Sciences" course et Università degli Studi of Insubria, Varese, Italy. No sponsorships or competing interests have been disclosed for this article.

Fabrizia Elli

fabrizia.elli@hotmail.it

1 Division of Otorhinolaryngology, Department of Biotechnology and Life Sciences, University of Insubria, Ospedale di Circolo e Fondazione Macchi, Via Guicciardini 9, 21100 Varese, Italy

2 Department of Biotechnology and Life Sciences, Head and Neck Surgery and Forensic Dissection Research Center (HNS\&FDRc), University of Insubria, Varese, Italy
A significant drop in visitations to the Emergency Department was observed during the last months of COVID-19 pandemic, probably due to both the lockdown imposed by Regional and National dispositions and concerns of a potential contagion among patients.

The experience of a tertiary-care ear, nose and throat (ENT) Emergency Department was described and analysed during the COVID-19 pandemic. The period considered in this study, ranging between February 24th and May 7th, 2020 , corresponds to the time laps between the spread of the virus in northern Italy and the end of the lockdown imposed by the Italian government.

\section{Methods}

A retrospective review of the activity of a tertiary-care ENT Department in northern Italy regarding emergency consultations was carried out. Data were retrieved by a dedicated electronic-based database, considering all the accesses to the Emergency Department requiring an ENT consultation, occurring between February 24th and May 7th, 2020. February 24 th was the starting point of the analysis because it 
was the first working day after the beginning of the outbreak in Italy, which corresponded to the report of the first Italian patient affected by SARS-CoV-2 infection in Codogno, Lombardy, disclosed on February 21 st. May 7 th was considered as the ending date of the analysis because it corresponds to the end of the travel restriction imposed by the Government. Both the number of consultations performed and the diagnosis provided or the conditions treated have been registered. The procedures were stratified in 1-week time spans, to evaluate the evolving trends during the ongoing epidemic. A comparison of the number of visitations performed during this period was carried out with the same periods of time of the two previous years.

\section{Results}

During the period considered, a total of 193 visitations to the Emergency Department were performed. A significant reduction was observed in comparison with the same period of the two previous years, as high as $-68.2 \%$ if compared with 2018 (607 consultations) and $62.16 \%$ if compared to
2019 (510 consultations). The most relevant reduction rate in accesses was observed between March 23rd and March 30th, 2020 ( $-89 \%$ compared to $2019,-91 \%$ compared to 2018), which corresponded to the epidemic peak in COVID19-positive cases in Lombardy (Fig. 1).

When analysing the conditions treated, the most relevant reduction regarded facial trauma $(-70 \%)$, followed by upper aero-digestive tract foreign bodies $(-68 \%)$, infective of inflammatory conditions (e.g. acute tonsillitis, otitis, pharyngitis) (-59\%), and epistaxis (-47\%) (Fig. 2).

\section{Discussion}

In late February 2020, Italy was the earliest hit European country by the COVID-19 pandemic. After few days of confusion, Regional Government disposed self-containment and lockdown of transportation, to contrast the outbreak. Meanwhile, hospital dispositions dictated suspension of all elective and deferrable activities, regarding both surgery [2] and outpatient clinic visitations [3]. During this period of time and until the end of May, only Emergency Department consultations and postoperative and oncologic controls were

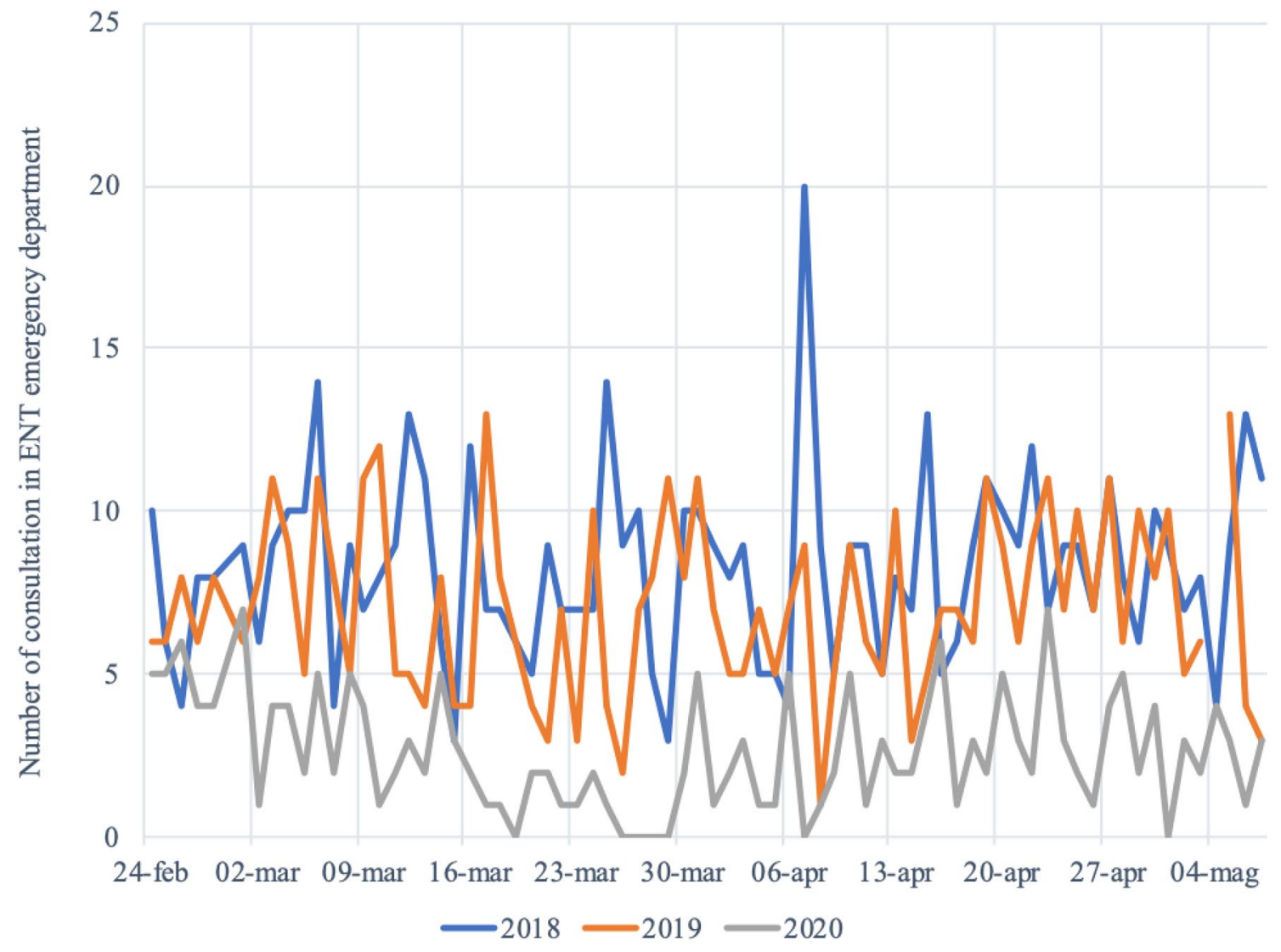

Fig. 1 Comparison between the number of consultation in the ENT Emergency Department in the last 2 years and in the pandemic period of COVID-19 (February 24th, 2020-May 7th, 2020) 


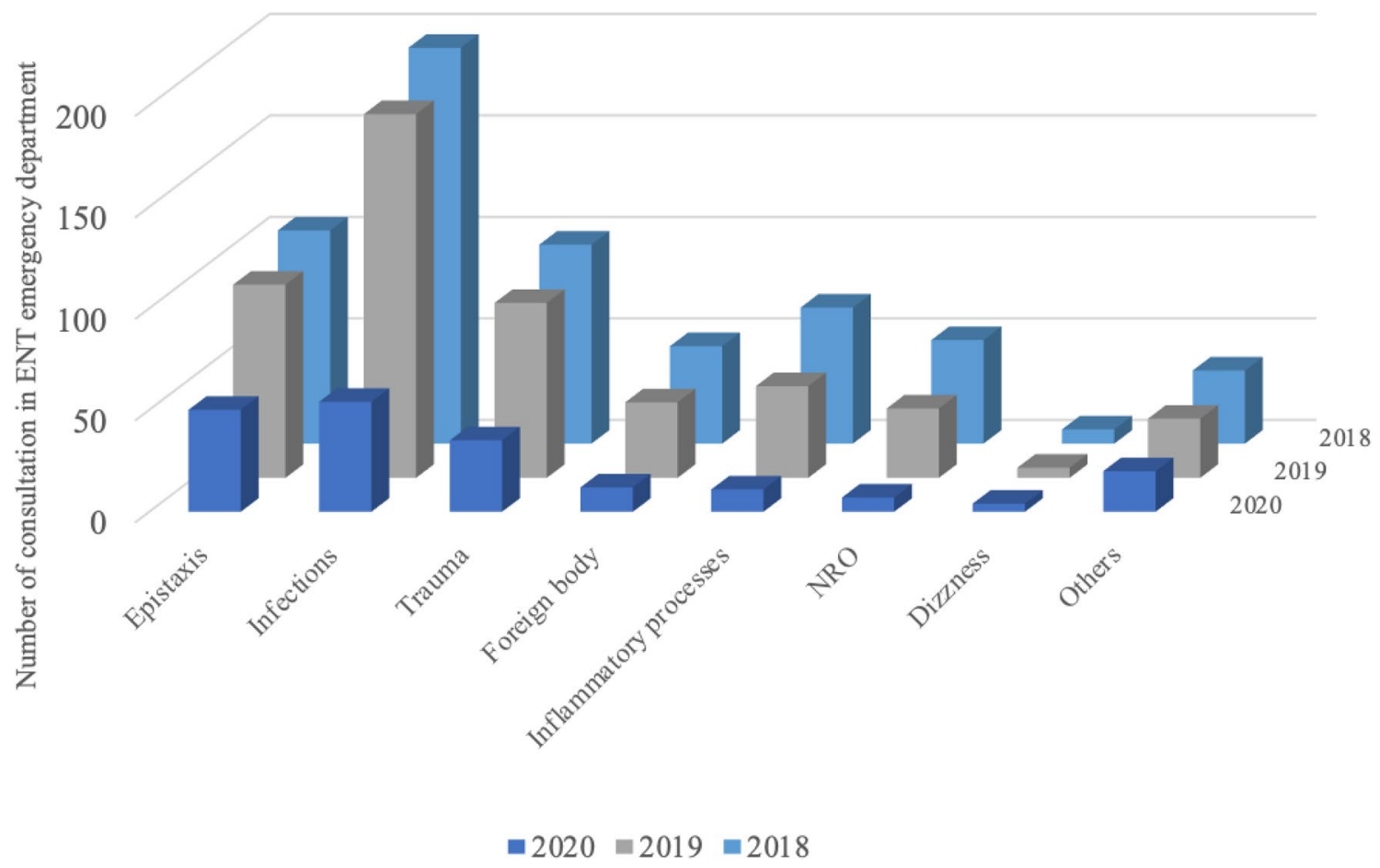

Fig. 2 Comparison between the number of consultation in the ENT Emergency Department according to the conditions treated and diagnosis provided in the last 2 years and in the pandemic period of COVID-19 (February 24th, 2020-May 7th, 2020)

guaranteed in our Department, according to dispositions provided and an internal reorganization based on the necessity for the Department to cope with the outbreak, while still providing adequate care for patients [4-6]. A significant reduction of the outpatient volume of consultation has already been reported by our group [3]. The analysis performed in the present paper highlights a contextual considerable drop in the number of Emergency Department consultations. The reduction in the activity was as high as $-89 \%$ compared to 2019 and $-91 \%$ compared to 2018 . The reasons for this are various. As regards traumatisms, for which a reduction of $70 \%$, if compared to the previous year, could be noticed, the fall in consultations is most probably related to the lockdown measures imposed by the Regional Government (e.g. closure of schools, ban on participating in team sports, restriction on personal transportation), which prevented the occurrence of traumatism itself. Epistaxis, on the other hand, was the disease for which the lowest reduction was observed in the comparison with previous years $(-47 \%)$, most probably due to the impossibility to defer a specialist ENT consultation in those cases of epistaxis not resolving spontaneously. As regards other conditions treated (inflammatory processes, nasal respiratory obstruction, hearing loss and dizziness), a significant reduction might be justified considering the diffuse fear of accessing the hospital, which was considered a risky place for possible contagion by SARS-Cov-2. This has probably induced many people to defer consultation to a latter period, once the rising epidemic concerns would diminish.

This marked reduction in emergency consultations was helpful to reduce the workload for the department in the time of crisis, in which most of the resources (healthcare staff, personal protective equipment and instrumentations) were employed to face the on-going epidemic. However, it is foreseeable to think that this situation will not last long if the reduction in COVID-19-positive cases continues, and so the burden of emergency consultations in the field of ENT might rise soon. The analysis performed might be useful as a starting point for a future reorganization of the management of first-aid consultations once the epidemic will be resolved.

The pandemic, even in its condition of social disaster and health emergency, can be used as a starting point to improve assistance and care for people for the future. The inappropriate access to the Emergency Department, used at any time of day to quickly obtain a specialist examination, must be discouraged, and it is advisable to readjust the methods of access to hospital care, to avoid abuse and misuse. The decrease in the waiting time for outpatient ENT specialist consultation with national healthcare system along with improvements in territorial medicine would be two practical ways to prevent inappropriate accesses to the Emergency Department. Leaving behind the COVID-19 outbreak, the 
hospital-centric health system should be gradually overcome by a new organization focused on territorial health community, able to take charge of the patient, and ensure, through dedicated facilities, continuity of care and integration with social welfare.

Author contributions All authors contributed to data analysis and manuscript writing. All authors have reviewed and approved the final version of the manuscript.

Funding Nothing to disclose.

Data availability Not applicable.

Code availability Not applicable.

\section{Compliance with ethical standards}

Conflict of interest Nothing to disclose.

\section{References}

1. John's Hopkins Center for Systems Science and Engineering. COVID-19 Dashboard by the Center for Systems Science and
Engineering (CSSE) at Johns Hopkins University (JHU). https:// coronavirus.jhu.edu/map.html. Accessed 31 May 2020

2. Castelnuovo P, Turri-Zanoni M, Karligkiotis A et al (2020) Skull base surgery during the Covid-19 pandemic: the Italian skull base society recommendations. Int Forum Allergy Rhinol. https://doi. org/10.1002/alr.22596

3. De Bernardi F, Turri-Zanoni M, Battaglia P, Castelnuovo P (2020) How to reorganize an ear, nose, and throat outpatient service during the COVID-19 outbreak: report from northern Italy. Laryngoscope. https://doi.org/10.1002/lary.28716

4. Turri-Zanoni M, Battaglia P, Karligkiotis A, Locatelli D, Castelnuovo P (2020) Managing care for patients with sinonasal and anterior skull base cancers during the COVID-19 pandemic. Head Neck. https://doi.org/10.1002/hed.26257

5. Karligkiotis A, Arosio AD, Battaglia P, Sileo G, Czaczkers C, Volpi L, Turri-Zanoni M, Castelnuovo P (2020) Changing paradigms in sinus and skull base surgery as the COVID-19 pandemic evolves: preliminary experience from a single Italian Tertiary Care Center. Head Neck. https://doi.org/10.1002/hed.26320

6. Karligkiotis A, Arosio AD, Castelnuovo P (2020) How to obtain a Nasopharyngeal Swab Specimen. N Engl J Med. https://doi. org/10.1056/NEJMc2015949

Publisher's Note Springer Nature remains neutral with regard to jurisdictional claims in published maps and institutional affiliations. 